\title{
Efficacy and safety of esaxerenone (CS-3150) for the treatment of essential hypertension: a phase 2 randomized, placebo-controlled, double-blind study
}

\author{
Sadayoshi Ito ${ }^{1} \cdot$ Hiroshi Itoh ${ }^{2} \cdot$ Hiromi Rakugi $\circledast^{3} \cdot$ Yasuyuki Okuda ${ }^{4} \cdot$ Satoru Yamakawa $^{4}$ \\ Received: 18 December 2018 / Revised: 25 March 2019 / Accepted: 8 April 2019 / Published online: 21 May 2019 \\ (c) Springer Nature Limited 2019. This article is published with open access
}

\begin{abstract}
This was a phase 2, multicenter, randomized, double-blind, placebo-controlled, open-label comparator study to investigate the efficacy and safety of esaxerenone (CS-3150), a novel non-steroidal mineralocorticoid receptor blocker, in Japanese patients with essential hypertension. Eligible patients $(n=426)$ received esaxerenone $(1.25,2.5$, or $5 \mathrm{mg} /$ day $)$, placebo, or eplerenone (50-100 mg/day) for 12 weeks. The primary efficacy endpoint was the change from baseline in sitting systolic and diastolic blood pressure (BP). Safety endpoints included adverse events and serum $\mathrm{K}^{+}$elevation. There were significant dose-response reductions in the 2.5 and $5 \mathrm{mg} /$ day esaxerenone groups for sitting BP (both $p<0.001$ ) and 24-h BP (both $p<$ $0.0001)$ compared with placebo, with a mean (95\% confidence interval) change in sitting BP of $-7.0(-9.5$ to -4.6$) /-3.8$ $(-5.2$ to -2.4$) \mathrm{mmHg}$ in the placebo group, and $-10.7(-13.2$ to -8.2$) /-5.0(-6.4$ to -3.6$) \mathrm{mmHg},-14.3(-16.8$ to -11.9$) /-7.6(-9.1$ to -6.2$) \mathrm{mmHg}$, and $-20.6(-23.0$ to -18.2$) /-10.4(-11.8$ to -9.0$) \mathrm{mmHg}$ for the $1.25,2.5$, and $5 \mathrm{mg} /$ day esaxerenone groups, respectively, while the change was $-17.4(-19.9$ to -15.0$) /-8.5(-9.9$ to -7.1$) \mathrm{mmHg}$ for eplerenone. The incidence of adverse events was similar in all treatment groups. Serum $\mathrm{K}^{+}$levels initially increased in proportion with esaxerenone dose but were stable from week 2 until week 12. Plasma esaxerenone concentration increased in proportion with the dose. In conclusion, esaxerenone is an effective and tolerable treatment option for patients with essential hypertension.
\end{abstract}

\section{Introduction}

Hypertension is a multifactorial disease involving complex interactions between various metabolic, neurohormonal, and

Supplementary information The online version of this article (https:// doi.org/10.1038/s41371-019-0207-x) contains supplementary material, which is available to authorized users.

Sadayoshi Ito

db554@med.tohoku.ac.jp

1 Division of Nephrology, Endocrinology and Vascular Medicine, Department of Medicine, Tohoku University School of Medicine, Sendai, Japan

2 Division of Endocrinology, Metabolism and Nephrology, Department of Internal Medicine, Keio University School of Medicine, Tokyo, Japan

3 Department of Geriatric and General Medicine, Osaka University Graduate School of Medicine, Suita, Japan

4 Daiichi Sankyo Co., Ltd., Tokyo, Japan inflammatory factors, and is a leading risk factor for cardiovascular morbidity and mortality [1, 2]. Uncontrolled hypertension is associated with vasculopathy, heart disease, cerebrovascular diseases, and nephropathy, all of which are classic manifestations of hypertensive end-organ damage [3].

In addition to non-pharmacological treatments, management of hypertension often requires pharmacotherapy with an antihypertensive agent, which is a proven approach for reducing cardiovascular morbidity and mortality [4]. Although monotherapy may be effective in some patients, failure to achieve the desired antihypertensive effect requires the concurrent use of multiple antihypertensive drugs as part of a multifactorial strategy [5, 6]. In fact, many patients require three or more antihypertensive drugs to achieve a blood pressure (BP) level of $<140 / 90 \mathrm{mmHg}$ [7]. Triple drug therapy combinations usually include a renin-angiotensin system inhibitor (angiotensin converting enzyme $[\mathrm{ACE}]$ inhibitors or angiotensin II-receptor blockers), a calcium channel blocker, and a diuretic.

The Japanese Guidelines for the Management of Hypertension (2014) recommend adding an aldosterone 
antagonist to the treatment regimen of patients with poorly controlled BP or resistant hypertension [8]. Aldosterone is a steroid hormone that regulates electrolyte homeostasis and $\mathrm{BP}$ via binding to the mineralocorticoid receptors (MR) in the distal tubule and collecting duct of the kidney [9]. Primary aldosteronism (PA) is caused by the excess production of aldosterone and is the most common cause of secondary hypertension as well as a common cause of antihypertensive treatment resistance [10-12]. Furthermore, aldosteroneinduced MR activation impairs insulin sensitivity and is associated with obesity and diabetes [13, 14]. Therefore, treatment-resistant hypertension in the presence of conditions such as obesity, diabetes, and chronic kidney disease (CKD) can occur despite normal plasma aldosterone concentrations (PAC). The role of pathological overstimulation of MR in the absence of high aldosterone levels has been indicated in cases of MR-associated hypertension [15].

Various studies have demonstrated the utility of steroidal MR antagonists, such as spironolactone and eplerenone, in the treatment of resistant hypertension [16-18]. When added to a renin-angiotensin system (RAS) blocker, steroidal MR antagonists further reduce proteinuria in patients with CKD from either diabetic or non-diabetic causes [19]. Therefore, adding an aldosterone antagonist to the treatment regimen of patients with poorly controlled BP, or treatmentresistant hypertension, is recommended in the Japanese Guidelines for the Management of Hypertension (2014) [8], the American College of Cardiology/American Heart Association 2017 guideline for high blood pressure in adults [20], and the 2018 European Society of Cardiology and the European Society of Hypertension guidelines for the management of arterial hypertension [21]. However, spironolactone has significant treatment-emergent side effects such as gynecomastia [8]. While eplerenone has an improved MR selectivity, it seems to be less potent when compared with spironolactone [22]. In general, MR antagonists elevate serum $\mathrm{K}^{+}$levels and, as such, eplerenone is contraindicated in diabetic patients with albuminuria [23].

Esaxerenone (CS-3150) is a novel oral, non-steroidal, selective MR blocker, which is highly potent and could be used to treat hypertension and cardiovascular and renal disorders [24-26]. In preclinical studies, esaxerenone inhibited BP elevation in deoxycorticosterone acetate/saltinduced hypertensive rats and in Dahl salt-sensitive hypertensive rats with an additional protective effect on the heart and kidneys [24, 25]. In a phase 1 study, the tolerability of esaxerenone was confirmed after single- and multiple-dose escalations in healthy Japanese subjects [26]. Therefore, this phase 2 study was designed to evaluate the antihypertensive efficacy and safety of esaxerenone, and to determine the optimal dose for lowering BP in Japanese patients with essential hypertension.

\section{Patients and methods}

\section{Ethics}

The study protocol was reviewed and approved by the independent institutional review board for each center. This study was conducted in adherence with the International Conference on Harmonization Guidelines for Good Clinical Practices, applicable local regulations, and the ethical principles based on the Declaration of Helsinki. All patients provided written informed consent prior to participation.

\section{Patients}

Patients were eligible for enrollment based on the key inclusion criteria: aged $\geq 20$ years at time of informed consent; sitting systolic BP (SBP) of $\geq 140$ to $<180 \mathrm{mmHg}$ and diastolic BP (DBP) $\geq 90$ to $<110 \mathrm{mmHg}$; and 24-h BP by ambulatory BP monitoring (ABPM) of $\geq 130 / 80 \mathrm{mmHg}$. The main exclusion criteria were secondary hypertension or malignant hypertension; diabetes with albuminuria; serum $\mathrm{K}^{+}$level $<3.5$ or $\geq 5.1 \mathrm{mEq} / \mathrm{L}$; and creatinine-adjusted estimated glomerular filtration rate (eGFRcreat) $<60 \mathrm{~mL} /$ $\min / 1.73 \mathrm{~m}^{2}$. Additional criteria are detailed in Supplementary Information 1.

\section{Study design}

This was a phase 2, multicenter, randomized, double-blind, placebo-controlled, open-label comparator study across 19 sites in Japan from January to September 2015. All patients underwent a 4-week screening period to remove the effects of prior therapeutic agents. During this period, patients received two placebo tablets administered orally once daily after breakfast.

Eligible patients were then randomized in a 1:1:1:1:1 ratio using stratification by baseline sitting $\operatorname{SBP}(<160, \geq 160)$ to the following treatment groups: $1.25 \mathrm{mg} /$ day esaxerenone (one 1.25-mg esaxerenone and one placebo tablet), $2.5 \mathrm{mg} /$ day esaxerenone (two 1.25-mg esaxerenone tablets), or $5 \mathrm{mg} /$ day esaxerenone (two 2.5-mg esaxerenone tablets), eplerenone or placebo (two placebo tablets) for 12 weeks. In the eplerenone group, patients were administered $50 \mathrm{mg} /$ day eplerenone for 2-4 weeks followed by $100 \mathrm{mg} /$ day of eplerenone for 8-10 weeks (one 50-mg tablet and two 50-mg eplerenone tablets, respectively).

Esaxerenone and the open-label comparator eplerenone were administered orally once daily after breakfast for 12 weeks. The treatment period was succeeded by a 2 -week follow-up period.

The criteria for withdrawal included: the presence of hyperkalemia defined as a serum $\mathrm{K}^{+}$level of $\geq 6.0 \mathrm{mEq} / \mathrm{L}$ or $\geq 5.5 \mathrm{mEq} / \mathrm{L}$ on two consecutive measurements; SBP and 
DBP persistently $<90 \mathrm{mmHg}$ and $<50 \mathrm{mmHg}$, respectively; SBP and DBP persistently $\geq 180 \mathrm{mmHg}$ and $\geq 110 \mathrm{mmHg}$, respectively; and eGFRcreat values of $<45 \mathrm{~mL} / \mathrm{min} / 1.73$ $\mathrm{m}^{2}$. These criteria were judged by an investigator or subinvestigator to determine whether the patient was to discontinue treatment.

\section{Prior and concomitant medications}

Prohibited concomitant drugs and therapies that could not be used from 4 weeks prior to the start of the treatment period to the completion of the study included antihypertensive drugs (angiotensin II receptor blockers, calcium channel blockers, ACE inhibitors, beta blockers [including alpha-beta blockers], alpha blockers, other sympatholytics, vasodilators, and renin inhibitors); diuretics (thiazide diuretics, thiazide analog diuretics, loop diuretics, and potassium-sparing diuretics); Chinese herbal medicines; cytochrome P450 3A4 inhibitors (itraconazole, ritonavir, nelfinavir mesilate, clarithromycin, and verapamil hydrochloride); $\mathrm{K}^{+}$preparations; and ion exchange resin.

Non-steroidal anti-inflammatory drugs were also prohibited from 4 weeks prior to the start of the treatment period to the completion of the study. However, they could be used for a maximum of 5 days in a row, as external preparations intended for a topical effect (except for suppositories), and aspirin could be used continuously at a daily dosage of $\leq 324 \mathrm{mg}$.

\section{BP measurements}

Sitting BP was measured at start, week 3 and the end of the observation period, at weeks $1,2,4,6,8,10$, and 12 of the treatment period, 1 day after the end of treatment, or at study discontinuation using an automatic BP monitor (HEM-759P Fuzzy device, Omron Healthcare Co., Ltd., Mukou, Japan). Each measurement was taken within 21-27 h after the study drug was administered. BP measurement was always performed prior to blood sampling when both were scheduled on the same day, and at least $3 \mathrm{~h}$ after a meal. For each time point, BP measurement was repeated three times with 1- to 2-min intervals following a resting period of at least $5 \mathrm{~min}$ while sitting.

In addition, 24-h BP was measured at week 3 of the observation period and week 12 of the treatment period using an ambulatory BP monitor (TM-2433, A \& D Co., Ltd., Tokyo, Japan). The study drug was administered after sitting BP measurements and prior to ABPM. BP measurements were taken over a period of at least $25 \mathrm{~h}$ with 30 min intervals. The automatic BP measurements and ABPM were operated by the doctors, nurses, or clinical laboratory technicians involved in this study.

\section{Efficacy analysis}

The primary efficacy endpoint was the change from baseline in sitting BP (SBP and DBP) at the end of the treatment period defined as the average sitting BP of week 10 and week 12 after last observation carried forward (LOCF) imputation of missing values. The sitting SBP and DBP (at trough) were measured at baseline and then on weeks 1,2 , $4,6,8,10$, and 12 . The secondary efficacy endpoint was the change from baseline in mean 24-h BP at week 12.

Other efficacy endpoints included change in sitting BP, change in diurnal, morning (06:00-08:59), daytime (07:00-21:59), and nocturnal (22:00-06:59) BP, the proportion of patients that achieved the sitting BP target $(<140 /$ $90 \mathrm{mmHg}$ ), and the proportion of patients that achieved a mean 24-h BP of <140/90 mmHg.

Subgroup analyses were performed to evaluate the antihypertensive effect (measured by sitting BP) of esaxerenone based on the following baseline factors: sitting BP (SBP and DBP), hypertension grade (Grade I or II) [8], PAC, plasma renin activity (PRA), eGFRcreat, serum $\mathrm{K}^{+}$, and the presence or absence of diabetes.

\section{Safety analysis}

Safety variables included adverse events (AEs), clinical laboratory tests (hematology and serum biochemistry), vital signs (sitting BP, pulse rate, and ABPM), body weight, 12lead electrocardiogram, and serum $\mathrm{K}^{+}$changes from baseline. Clinical laboratory tests and 12-lead electrocardiogram were performed at baseline and on weeks 4,8 , and 12 during the treatment period. Vital signs (except ABPM) were measured at baseline and on weeks 1, 2, 4, 6, 8, 10, 12, and the day after the week 12 visit. Body weight was measured at baseline and the day after the week 12 visit. In addition, serum $\mathrm{K}^{+}$was measured on weeks $1,2,4,6,8,10$, and 12 , and 2 weeks after the end of the treatment.

All the details of AEs and side effects of the study drug have been reported to the drug manufacturer, Daiichi Sankyo Co., Ltd., in the clinical study report document (reference number: CS3150-A-J203).

\section{Pharmacokinetic analysis}

Plasma esaxerenone trough concentrations $\left(\mathrm{C}_{\text {trough }}\right)$ were measured from blood samples collected on weeks 4 and 12 . The method for blood sample analysis has been described previously [26].

\section{Analysis of PAC and PRA}

The renin-angiotensin-aldosterone system (RAAS) hormones, including PAC and PRA. PAC was measured using 
a radioimmunoassay and PRA was measured using an enzyme immune assay on blood samples collected on weeks 4 and 12. The methods used to measure PAC and PRA have been described previously [26].

\section{Statistical analyses}

The efficacy analysis was conducted in the full analysis set (FAS), containing patients who were treated with the study drug at least once and for whom measurements were taken for at least one variable pertaining to efficacy after the start of treatment. Safety was assessed in the safety analysis set (SAS), comprising all patients who received at least one dose of the study drug.

The primary efficacy analysis was the change in sitting BP from baseline and was calculated for each treatment group (least squares [LS] mean) with corresponding 95\% confidence intervals (CIs) using an analysis of covariance (ANCOVA) model, with change from baseline at end of treatment as the objective variable, treatment group as the explanatory variable, and baseline $\mathrm{BP}$ as the covariate. The same ANCOVA model was also used to compare each esaxerenone dosing group with the placebo group. The difference in LS means, corresponding 95\% CIs, and $P$ values were also calculated. To adjust the multiplicity of statistical tests, the fixed sequence procedure was applied: the comparison was initially conducted between the higherdose esaxerenone groups and the placebo group with twosided 5\% significance levels, the comparison for the lower dosage groups was continued at a $5 \%$ significance level in descending order of dosage but only when significance was shown in the higher dose.

The analysis performed on the primary efficacy endpoint was repeated for the difference in 24-h BP measurements at screening period week 3 and treatment period week 12 .

For the remaining efficacy endpoints (change in sitting $\mathrm{BP}$ at each visit, diurnal and nocturnal BP [systolic, diastolic, and mean]), summary statistics were calculated by treatment group for each time point and change from baseline. The same ANCOVA model as the primary endpoint was also used to calculate point estimates of change and corresponding 95\% CIs by treatment group for each measured time point and to plot trend graphs. For the subgroup analyses, the same statistical model for the primary efficacy endpoint was applied to all subgroups based on sitting BP (SBP/DBP).

Summary statistics were calculated for PAC and PRA by treatment group for each time point. The adjusted LS geometric mean and corresponding 95\% CIs were calculated for percentage change in each group at each time point using an ANCOVA model, with change from baseline at each time point an objective variable, treatment group an explanatory variable, and screening period data. Summary statistics were also calculated for determining the pharmacokinetics of plasma esaxerenone concentrations at each time point at weeks 4 and 12. Safety analyses were conducted in a descriptive manner and presented with the appropriate summary statistics by treatment group. All statistical analyses were performed using SAS 9.3 (SAS Institute, Cary, NC).

\section{Results}

\section{Patient disposition}

Of the 687 patients who provided written consent, 426 met the inclusion criteria and were randomly assigned to one of the study groups: placebo $(n=87)$, esaxerenone $1.25 \mathrm{mg}$ / day $(n=83)$, esaxerenone $2.5 \mathrm{mg} /$ day $(n=84)$, esaxerenone $5 \mathrm{mg} /$ day $(n=88)$, and eplerenone $(n=84)$. In the eplerenone group, treatment began at $50 \mathrm{mg} /$ day and was increased to $100 \mathrm{mg} /$ day in $78.6 \%$ (66/84 patients) of patients by week 2 and $89.3 \%$ (75/84 patients) of patients by week 4. After week 4, no further dose changes were made in the eplerenone group.

All 426 randomized patients were included in the SAS, 423 had efficacy data and were included in the FAS, and 403 patients completed the study (Fig. 1). Of the 23 patients who discontinued the study, 13 withdrew by their own choice, four because of AEs, one was lost to follow-up, four met the criterion for withdrawal (both SBP and DBP persistently $\geq 180 \mathrm{mmHg}$ and $\geq 110 \mathrm{mmHg}$, respectively), and one was suspended by the investigators due to high BP.

Patient demographics are shown in Table 1. In brief, $69.7 \%$ of patients were male, the mean age was 57.0 years, sitting SBP/DBP at baseline was $157.0 / 97.6 \mathrm{mmHg}$, and $52.2 \%$ of patients had received prior treatment for hypertension. There were no remarkable differences between treatment groups.

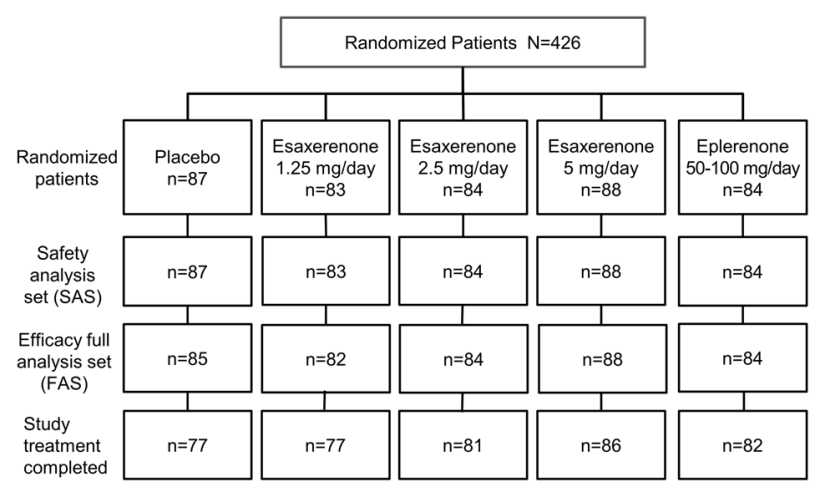

Fig. 1 Patient disposition 
Table 1 Patient demographics (full analysis set)

\begin{tabular}{|c|c|c|c|c|c|c|}
\hline & $\begin{array}{c}\text { Placebo } \\
n=85\end{array}$ & $\begin{array}{l}\text { Esaxerenone } \\
1.25 \mathrm{mg} / \text { day } \\
n=82\end{array}$ & $\begin{array}{l}\text { Esaxerenone } \\
2.5 \mathrm{mg} / \text { day } \\
n=84\end{array}$ & $\begin{array}{l}\text { Esaxerenone } \\
5 \mathrm{mg} / \text { day } \\
n=88\end{array}$ & $\begin{array}{l}\text { Eplerenone } \\
50-100 \mathrm{mg} / \text { day } \\
n=84\end{array}$ & $\begin{array}{l}\text { All } \\
N=423\end{array}$ \\
\hline Sex (male), $n(\%)$ & $60(70.6)$ & $55(67.1)$ & $54(64.3)$ & $65(73.9)$ & $61(72.6)$ & $295(69.7)$ \\
\hline Age (years) & $57.3 \pm 9.1$ & $57.2 \pm 9.3$ & $56.8 \pm 9.4$ & $57.1 \pm 8.8$ & $56.5 \pm 10.0$ & $57.0 \pm 9.3$ \\
\hline Weight (kg) & $69.0 \pm 13.5$ & $68.8 \pm 12.3$ & $67.9 \pm 12.1$ & $69.8 \pm 13.2$ & $70.7 \pm 17.0$ & $69.3 \pm 13.7$ \\
\hline BMI $\left(\mathrm{kg} / \mathrm{m}^{2}\right)$ & $25.5 \pm 4.1$ & $25.3 \pm 3.7$ & $24.9 \pm 3.3$ & $25.7 \pm 3.7$ & $25.8 \pm 4.9$ & $25.5 \pm 4.0$ \\
\hline SBP (sitting, mmHg) & $156.7 \pm 9.0$ & $156.4 \pm 9.1$ & $156.4 \pm 8.4$ & $157.4 \pm 9.0$ & $157.9 \pm 8.4$ & $157.0 \pm 8.8$ \\
\hline DBP (sitting, mmHg) & $96.8 \pm 5.0$ & $97.2 \pm 5.5$ & $98.6 \pm 5.6$ & $97.2 \pm 5.4$ & $98.4 \pm 5.3$ & $97.6 \pm 5.4$ \\
\hline SBP (ABPM, mmHg) & $167.0 \pm 12.1$ & $166.2 \pm 14.7$ & $165.0 \pm 15.5$ & $167.1 \pm 15.3$ & $165.9 \pm 14.0$ & $166.2 \pm 14.3$ \\
\hline DBP (ABPM, mmHg) & $97.9 \pm 7.6$ & $98.9 \pm 9.0$ & $98.9 \pm 10.0$ & $98.5 \pm 7.2$ & $98.3 \pm 8.0$ & $98.5 \pm 8.3$ \\
\hline Pulse rate (bpm) & $72.1 \pm 9.6$ & $73.3 \pm 10.2$ & $73.3 \pm 9.5$ & $71.7 \pm 9.2$ & $73.8 \pm 10.2$ & $72.8 \pm 9.7$ \\
\hline $\begin{array}{l}\text { Prior treatment for } \\
\text { hypertension }^{\mathrm{a}}, n(\%)\end{array}$ & $44(51.8)$ & $43(52.4)$ & $43(51.2)$ & $50(56.8)$ & $41(48.8)$ & $221(52.2)$ \\
\hline Presence of diabetes, $n(\%)$ & $9(10.6)$ & $11(13.4)$ & $8(9.5)$ & $10(11.4)$ & $20(23.8)$ & $58(13.7)$ \\
\hline LDL cholesterol (mg/dL) & $129.1 \pm 32.6$ & $127.5 \pm 31.0$ & $130.4 \pm 31.3$ & $132.0 \pm 34.4$ & $127.2 \pm 30.2$ & $129.3 \pm 31.8$ \\
\hline Serum $\mathrm{K}^{+}(\mathrm{mEq} / \mathrm{L})$ & $4.14 \pm 0.31$ & $4.07 \pm 0.28$ & $4.10 \pm 0.25$ & $4.14 \pm 0.29$ & $4.09 \pm 0.28$ & $4.11 \pm 0.29$ \\
\hline $\mathrm{HbA1c}(\%)$ & $5.64 \pm 0.59$ & $5.67 \pm 0.65$ & $5.62 \pm 0.63$ & $5.46 \pm 0.42$ & $5.76 \pm 0.63$ & $5.63 \pm 0.60$ \\
\hline FPG (mg/dL) & $106.3 \pm 16.4$ & $109.7 \pm 21.2$ & $105.9 \pm 17.3$ & $104.2 \pm 14.0$ & $109.5 \pm 19.9$ & $107.1 \pm 17.9$ \\
\hline $\begin{array}{l}\text { eGFRcreat }(\mathrm{mL} / \mathrm{min} / \\
\left.1.73 \mathrm{~m}^{2}\right)\end{array}$ & $78.0 \pm 11.6$ & $77.0 \pm 12.2$ & $80.3 \pm 11.9$ & $79.6 \pm 11.5$ & $81.3 \pm 12.2$ & $79.2 \pm 11.9$ \\
\hline PRA (ng/mL/h) & $1.05 \pm 0.91$ & $1.10 \pm 1.05$ & $1.11 \pm 1.00$ & $0.96 \pm 1.09$ & $1.09 \pm 0.97$ & $1.06 \pm 1.00$ \\
\hline $\mathrm{PAC}(\mathrm{pg} / \mathrm{mL})$ & $116.5 \pm 50.15$ & $112.8 \pm 35.68$ & $110.0 \pm 37.32$ & $107.5 \pm 42.51$ & $113.7 \pm 39.85$ & $112.1 \pm 41.37$ \\
\hline \multicolumn{7}{|l|}{ Alcohol consumption, $n(\%)$} \\
\hline Never & $20(23.5)$ & $24(29.3)$ & $23(27.4)$ & $21(23.9)$ & $25(29.8)$ & $113(26.7)$ \\
\hline Former & $7(8.2)$ & $6(7.3)$ & $3(3.6)$ & $6(6.8)$ & $2(2.4)$ & $24(5.7)$ \\
\hline Current & $58(68.2)$ & $52(63.4)$ & $58(69.0)$ & $61(69.3)$ & 57 (67.9) & $286(67.6)$ \\
\hline
\end{tabular}

Data are presented as mean $\pm \mathrm{SD}$, unless otherwise stated

$A B P M$ ambulatory BP monitoring, $D B P$ diastolic blood pressure, eGFRcreat estimated glomerular filtration rate with creatinine, $F P G$ fasting plasma glucose, $H b A 1 c$ hemoglobin A1c, $L D L$ low-density lipoprotein, $P R A$ plasma renin activity, $P A C$ plasma aldosterone concentration, $S B P$ systolic blood pressure

${ }^{\mathrm{a}}$ Within 4 weeks prior to run-in period

\section{Efficacy analysis}

The mean changes from baseline in sitting BP at the end of treatment are shown in Figs 2 and 3. There was a clear dose-response relationship for BP reduction. ANCOVA showed significant reductions in sitting SBP and DBP in the $2.5 \mathrm{mg} /$ day and $5 \mathrm{mg} /$ day esaxerenone groups compared with placebo (all $p<0.001$ ). At the end of the study, the LS mean (with 95\% CI) change in BP (LOCF) was -7.0 ( -9.5 to $-4.6) /-3.8(-5.2$ to -2.4$) \mathrm{mmHg}$ in the placebo group, and $-10.7(-13.2$ to -8.2$) /-5.0(-6.4$ to -3.6$) \mathrm{mmHg},-14.3$ $(-16.8$ to -11.9$) /-7.6(-9.1$ to -6.2$) \mathrm{mmHg}$, and -20.6 $(-23.0$ to -18.2$) /-10.4(-11.8$ to -9.0$) \mathrm{mmHg}$ for the 1.25 , 2.5 , and $5 \mathrm{mg}$ /day esaxerenone groups, respectively. In comparison, eplerenone therapy produced a BP change of -17.4 $(-19.9$ to -15.0$) /-8.5$ ( -9.9 to -7.1$) \mathrm{mmHg}$ after 12 weeks.

Similarly, 24-h BP changes showed a clear doseresponse relationship, and all esaxerenone doses

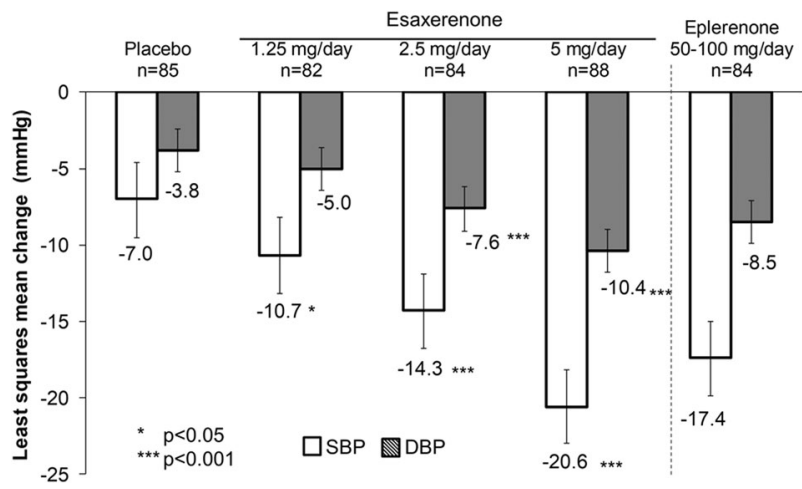

Fig. 2 Least square mean change from baseline in sitting systolic and diastolic blood pressures (full analysis set). SBP systolic blood pressure, DBP diastolic blood pressure

significantly lowered 24-h BP compared with placebo $(1.25 \mathrm{mg} / \mathrm{day}: p=0.0038$ and $p=0.0154$ for $24-\mathrm{h}$ SBP and DBP, respectively; 2.5 and $5 \mathrm{mg} /$ day: all $p<0.0001$ ) 


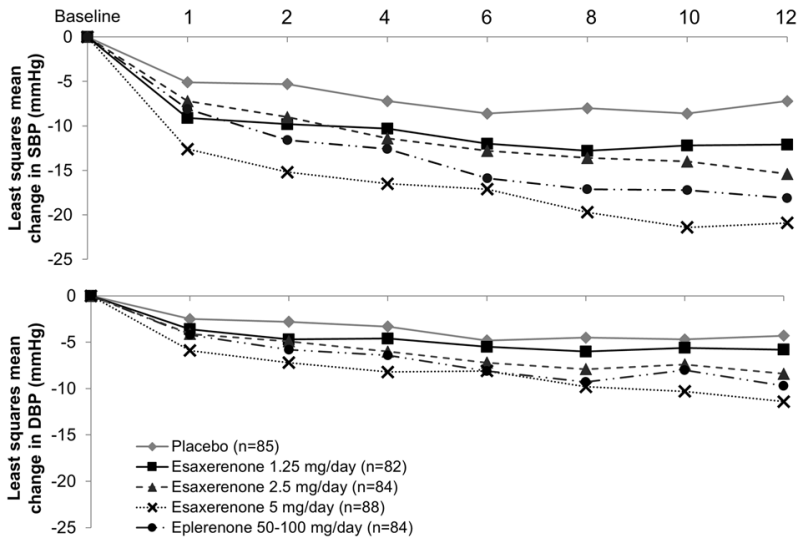

Fig. 3 Least square mean change from baseline in sitting systolic and diastolic blood pressures until the end of treatment (full analysis set). SBP systolic blood pressure, DBP diastolic blood pressure

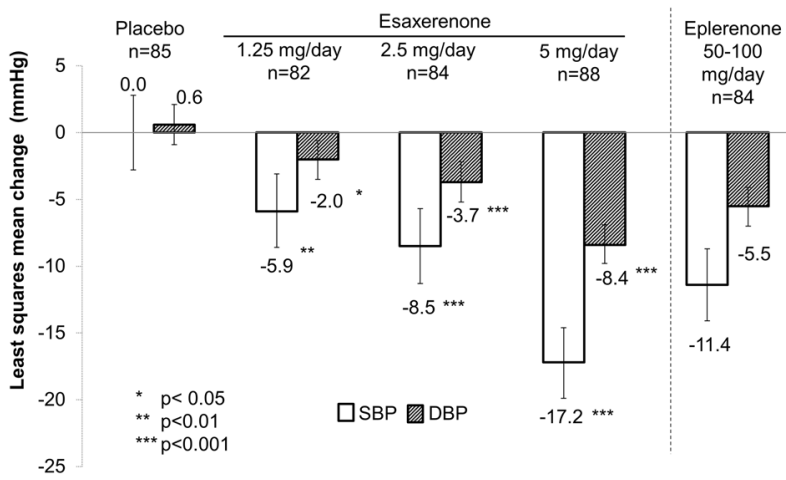

Fig. 4 Least square mean change from baseline in 24-h average ambulatory blood pressure (full analysis set). SBP systolic blood pressure, DBP diastolic blood pressure

(Fig. 4). The LS mean changes from baseline in morning, daytime, and nocturnal BP were greater in the esaxerenone groups compared with placebo (Supplementary Table 1). In addition, the LS mean changes from baseline in morning, daytime, and nocturnal BP after eplerenone treatment were comparable between the $2.5 \mathrm{mg} / \mathrm{day}$ esaxerenone and $5 \mathrm{mg} /$ day esaxerenone groups (data not shown).

The proportions of patients achieving a target BP of $<140 / 90 \mathrm{mmHg}$ at the end of esaxerenone treatment (LOCF) were $25.6 \%, 36.9 \%$, and $53.4 \%$ for the $1.25,2.5$, and $5 \mathrm{mg}$ /day groups, respectively (Fig. 5). In comparison, the proportions of patients achieving the same target $\mathrm{BP}$ were $17.6 \%$ in the placebo group and $34.5 \%$ in the eplerenone 50-100 mg group (Fig. 5).

Subgroup analyses were carried out based on the antihypertensive effect of esaxerenone on sitting BP when stratified by key patient demographics at baseline (Supplementary Table 2). Regardless of patients' baseline SBP/DBP, grade of hypertension, PAC, PRA, eGFRcreat, serum $\mathrm{K}^{+}$levels, or the presence or absence of diabetes

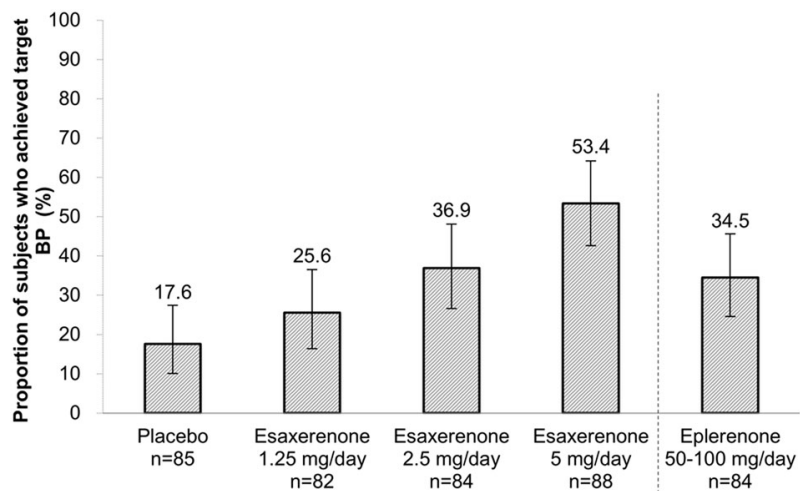

Fig. 5 Achievement rate of the target systolic and diastolic blood pressures (full analysis set). Target BP: both $\mathrm{SBP}<140 \mathrm{mmHg}$ and DBP $<90 \mathrm{mmHg}$. SBP systolic blood pressure, DBP diastolic blood pressure

mellitus, there was an antihypertensive effect across all subgroups.

\section{Safety analysis}

Safety analysis showed there were no marked differences in the incidence of AEs observed between patients taking placebo (46.0\% [40/87]), esaxerenone $1.25 \mathrm{mg} /$ day $(30.1 \%$ [25/ 83]), esaxerenone $2.5 \mathrm{mg} /$ day (40.5\% [34/84]), esaxerenone $5 \mathrm{mg} /$ day $(36.4 \%$ [32/88]), or eplerenone $50-100 \mathrm{mg} /$ day (36.9\% [31/84]) (Table 2). All AEs were determined to be either mild or moderate in severity. The most commonly observed AEs, with an incidence of $\geq 3.0 \%$ in any treatment group, were nasopharyngitis, upper respiratory tract inflammation, pharyngitis, headache, blood $\mathrm{K}^{+}$increased, blood uric acid increased, blood triglycerides increased, eGFRcreat decreased, back pain, blood creatine phosphokinase increased, and musculoskeletal stiffness. In addition, no AEs occurred that were considered related to sex hormones.

Serious AEs occurred in three patients during the treatment period, but only one of these patients (emergency hypertension) was from an esaxerenone treatment group $(1.25 \mathrm{mg} /$ day $)$. Although this patient was withdrawn from the study, a causal relationship with the study drug was ruled out. However, one patient was withdrawn from the eplerenone group due to a drug-related $\mathrm{AE}$ (diarrhea). No AEs were clinically significant, and no notable changes were observed in vital signs or body weight.

The change from baseline of serum $\mathrm{K}^{+}$increased according to the dose of esaxerenone administered. Serum $\mathrm{K}^{+}$increased to its highest value at weeks 1 and 2, and then reached steady state with a slight decrease over the course of the study (Figures $\mathrm{S} 1$ and $\mathrm{S} 2$ ). Hyperkalemia predefined as a serum $\mathrm{K}^{+}$level of $\geq 6.0 \mathrm{mEq} / \mathrm{L}$ or $\geq 5.5 \mathrm{mEq} / \mathrm{L}$ on two consecutive measurements was observed in one patient treated with esaxerenone $5 \mathrm{mg} /$ day (serum $\mathrm{K}^{+}: 4.4 \mathrm{mEq} / \mathrm{L}$ at baseline and $6.0 \mathrm{mEq} / \mathrm{L}$ 
Table 2 Adverse events (safety analysis set)

\begin{tabular}{|c|c|c|c|c|c|c|}
\hline & $\begin{array}{c}\text { Placebo } \\
n=87\end{array}$ & $\begin{array}{l}\text { Esaxerenone } \\
1.25 \mathrm{mg} / \text { day } \\
n=83\end{array}$ & $\begin{array}{l}\text { Esaxerenone } \\
2.5 \mathrm{mg} / \text { day } \\
n=84\end{array}$ & $\begin{array}{l}\text { Esaxerenone } \\
5 \mathrm{mg} / \text { day } \\
n=88\end{array}$ & $\begin{array}{l}\text { Eplerenone } \\
50-100 \mathrm{mg} / \text { day } \\
n=84\end{array}$ & $\begin{array}{l}\text { All } \\
N=426\end{array}$ \\
\hline Any adverse event & $40(46.0)$ & $25(30.1)$ & $34(40.5)$ & $32(36.4)$ & $31(36.9)$ & $162(38.0)$ \\
\hline Any serious adverse event & $2(2.3)$ & $1(1.2)$ & $0(0.0)$ & $0(0.0)$ & $0(0.0)$ & $3(0.7)$ \\
\hline Any drug-related adverse event & $8(9.2)$ & $8(9.6)$ & $7(8.3)$ & $12(13.6)$ & $7(8.3)$ & $42(9.9)$ \\
\hline Any drug-related serious adverse event & $0(0.0)$ & $0(0.0)$ & $0(0.0)$ & $0(0.0)$ & $0(0.0)$ & $0(0.0)$ \\
\hline $\begin{array}{l}\text { Number of patients who are } \\
\text { discontinued from the study due to } \\
\text { drug-related TEAE }\end{array}$ & $0(0.0)$ & $0(0.0)$ & $0(0.0)$ & $0(0.0)$ & $1(1.2)$ & $1(0.2)$ \\
\hline $\begin{array}{l}\text { Number of patients who discontinued } \\
\text { from the study due to hyperkalemia }\end{array}$ & $0(0.0)$ & $0(0.0)$ & $0(0.0)$ & $0(0.0)$ & $0(0.0)$ & $0(0.0)$ \\
\hline \multicolumn{7}{|c|}{ Adverse events occurring in $\geq 3 \%$ patients for any group } \\
\hline Nasopharyngitis & $7(8.0)$ & $4(4.8)$ & $6(7.1)$ & $6(6.8)$ & $7(8.3)$ & $30(7.0)$ \\
\hline Upper respiratory tract inflammation & $4(4.6)$ & $3(3.6)$ & $4(4.8)$ & $5(5.7)$ & $2(2.4)$ & $18(4.2)$ \\
\hline Pharyngitis & $2(2.3)$ & $3(3.6)$ & $5(6.0)$ & $1(1.1)$ & $2(2.4)$ & $13(3.1)$ \\
\hline Headache & $2(2.3)$ & $1(1.2)$ & $6(7.1)$ & $0(0.0)$ & $2(2.4)$ & $11(2.6)$ \\
\hline Back pain & $3(3.4)$ & $1(1.2)$ & $1(1.2)$ & $1(1.1)$ & $0(0.0)$ & $6(1.4)$ \\
\hline Musculoskeletal stiffness & $0(0.0)$ & $0(0.0)$ & $3(3.6)$ & $0(0.0)$ & $0(0.0)$ & $3(0.7)$ \\
\hline $\begin{array}{l}\text { Blood creatine phosphokinase } \\
\text { increased }\end{array}$ & $3(3.4)$ & $0(0.0)$ & $1(1.2)$ & $0(0.0)$ & $0(0.0)$ & $4(0.9)$ \\
\hline Blood $\mathrm{K}^{+}$increased & $2(2.3)$ & $0(0.0)$ & $3(3.6)$ & $3(3.4)$ & $1(1.2)$ & $9(2.1)$ \\
\hline Blood uric acid increased & $1(1.1)$ & $2(2.4)$ & $1(1.2)$ & $3(3.4)$ & $1(1.2)$ & $8(1.9)$ \\
\hline Blood triglycerides increased & $0(0.0)$ & $2(2.4)$ & $2(2.4)$ & $0(0.0)$ & $3(3.6)$ & $7(1.6)$ \\
\hline eGFRcreat decreased & $1(1.1)$ & $3(3.6)$ & $0(0.0)$ & $3(3.4)$ & $0(0.0)$ & $7(1.6)$ \\
\hline
\end{tabular}

Data are presented as $n(\%)$. System Organ Classes and Preferred Terms coded using MedDRA/J version 18.0. Percentages calculated using the number of subjects in the column heading as the denominator

$T E A E$ treatment-emergent adverse event, GFR glomerular filtration rate

measured once at week 12), however, this promptly recovered to $4.7 \mathrm{mEq} / \mathrm{L}$ on the next day. No patients were withdrawn from the study due to increased serum $\mathrm{K}^{+}$levels.

The mean (SD) changes from baseline in eGFRcreat at week 12 in the $1.25,2.5$, and $5 \mathrm{mg}$ /day esaxerenone groups were -2.31 (6.85), -3.69 (7.98), and -6.36 (8.08) $\mathrm{mL} /$ $\min / 1.73 \mathrm{~m}^{2}$, respectively. In comparison, the mean (SD) changes from baseline in eGFRcreat at week 12 for the placebo and eplerenone groups were 0.06 (6.05) and -2.11 (6.35) $\mathrm{mL} / \mathrm{min} / 1.73 \mathrm{~m}^{2}$, respectively.

\section{Pharmacokinetic analysis}

Plasma esaxerenone concentration $\left(\mathrm{C}_{\text {trough }}\right)$ levels generally increased in proportion with increasing esaxerenone dose and were similar at weeks 4 and 12 . At week 4 , the mean (SD) esaxerenone concentration levels were 8.61 (2.97), 16.39 (6.68), and $32.77(17.6) \mathrm{ng} / \mathrm{mL}$ in the $1.25,2.5$, and 5 $\mathrm{mg}$ /day groups, respectively. At week 12, the mean (SD) esaxerenone concentrations levels were 8.67 (2.87), 15.72 (6.74), and 32.74 (15.5) ng/mL in the $1.25,2.5$, and $5 \mathrm{mg} /$ day groups, respectively.

\section{Analysis of PAC and PRA}

PAC and PRA did not change in the placebo group; however, they increased in proportion with increasing esaxerenone dose. The mean percent $(95 \% \mathrm{CI})$ changes in PAC values from baseline until week 12 were $0.0 \%$ ( -8.0 to 8.6 ), $18.3 \%$ (8.8 to 28.5 ), $30.3 \%$ (20.1 to 41.3 ), $35.5 \%$ (25.2 to 46.6 ), and $19.5 \%$ (10.2 to 29.5 ) in the placebo group, the esaxerenone $1.25,2.5$, and $5 \mathrm{mg} /$ day groups, and the eplerenone group, respectively. The mean percent $(95 \% \mathrm{CI})$ changes in PRA from baseline until week 12 were $-5.5 \%$ ( -18.9 to 10.1 ), $27.4 \%$ (9.2 to 48.7 ), $44.4 \%$ (24.2 to 67.8 ), $119.6 \%$ (90.0 to 153.7 ), and $54.5 \%$ (33.3 to 79.1 ) in the placebo group, the esaxerenone $1.25,2.5$, and $5 \mathrm{mg} /$ day groups, and the eplerenone group, respectively.

\section{Discussion}

This multicenter study evaluated the antihypertensive effect and safety of esaxerenone, a novel non-steroidal MR blocker. The aim was to determine the optimal dose that 
would lower BP in Japanese patients with essential hypertension. We demonstrated a dose-response relationship in sitting and 24-h BPs, after 12 weeks of esaxerenone treatment.

Efficacy was evidenced by significant decreases in sitting BP at esaxerenone dosages of 2.5 and $5 \mathrm{mg}$ /day compared with placebo. There was also a significant difference in 24-h $\mathrm{BP}$ for all dosages of esaxerenone (1.25, 2.5, and $5 \mathrm{mg}$ /day) compared with placebo. In comparison, the antihypertensive effect of eplerenone was extrapolated to be between the data sets in the esaxerenone 2.5 and $5 \mathrm{mg} /$ day groups, which suggest that esaxerenone at $2.5-5 \mathrm{mg} /$ day has a similar antihypertensive effect to the clinical dosages of eplerenone $50-100 \mathrm{mg} /$ day.

Previous non-clinical studies have shown that esaxerenone has no agonistic or antagonistic effects on glucocorticoid, progesterone, or androgen receptors [24, 25]. The present clinical study therefore supports this conclusion as evidenced by the absence of sex hormone-related AEs.

In this study, there were no clear differences in the risk of increased serum $\mathrm{K}^{+}$levels when compared with placebo. Although the change in serum $\mathrm{K}^{+}$generally increased in proportion with esaxerenone dose and reached a maximum value at week 1 or week 2 of treatment, this increase did not continue through to week 12 . The mean (SD) baseline serum $\mathrm{K}^{+}$level for all patients was $4.11(0.29)$ and these levels remained stable throughout the study with maximum mean (SD) differences of 0.21 (0.31), 0.27 (0.26), and 0.33 $(0.31) \mathrm{mEq} / \mathrm{L}$ for the $1.25,2.5$, and $5 \mathrm{mg} /$ day esaxerenone groups, respectively. In most patients, serum $\mathrm{K}^{+}$levels did not reach $\geq 5.5 \mathrm{mEq} / \mathrm{L}$. Hyperkalemia was only detected in one patient in the esaxerenone $5 \mathrm{mg}$ /day group, however, this was transient and this patient recovered without further treatment. Hyperkalemia, a clinically relevant condition, is often a dose-response side effect of aldosterone antagonists such as spironolactone and eplerenone for the treatment of hypertension [27-30].

In the present study, esaxerenone plasma concentration increased in proportion with dose and was shown to be stable based on the similar values at week 4 and week 12 . As esaxerenone dose increased, the RAAS hormone measurements represented by PAC and PRA also increased in proportion, which is indicative of an MR antagonistic effect after oral administration of esaxerenone for 12 weeks. These changes in RAAS hormones were almost identical to that observed in the previous 6-week exploratory study (not published).

In the subgroup analysis, there were reductions in sitting BP even in patients with diabetes. There were also reductions in sitting BP (SBP/DBP) across a broad range of patient demographics. Analysis of the mean change in sitting BP from baseline to the end of the study showed that differences in the baseline hypertension grade, PAC, PRA,
eGFRcreat levels and serum $\mathrm{K}^{+}$levels had no clear effect on BP-lowering effects of esaxerenone therapy.

The present findings indicate that 2.5 and $5 \mathrm{mg} /$ day esaxerenone are optimal dosages based on the efficacy and safety data presented. The perceived limitations of this study include the small sample size, short observation period (12 weeks), and open-label nature of the comparator eplerenone. However, a randomized, double-blind, longterm phase 3 study has been completed recently to further investigate the safety and efficacy of esaxerenone therapy in essential hypertension patients. In addition, the safety and efficacy profiles of esaxerenone therapy in other patient populations, such as those affected by CKD and type 2 diabetes mellitus with albuminuria, have been assessed in several phase 3 studies that have been completed recently.

Esaxerenone is a novel non-steroidal MR blocker that has a dose-dependent antihypertensive effect on sitting SBP/ DBP and 24-h BP. Esaxerenone showed good efficacy and safety profiles in Japanese essential hypertensive patients, and no obvious safety concerns, including hyperkalemia, were observed across all doses of esaxerenone. In conclusion, esaxerenone 2.5 and $5 \mathrm{mg} /$ day are both considered optimum dosages for the treatment of essential hypertension.

\section{Summary Table}

\section{What is known about the topic?}

- Essential hypertension and end-organ damage are leading risk factors for morbidity and mortality throughout the world.

- Current guidelines recommend combination therapies that have complementary mechanisms to reduce blood pressure. Mineralocorticoid receptor (MR) antagonists are recommended for the treatment of resistant hypertension.

- An existing MR antagonist, spironolactone, has significant treatment-emergent side effects, which affect compliance rates. Eplerenone has an improved safety profile but may have less potent antihypertensive efficacy as compared with spironolactone.

\section{What this study adds}

- Esaxerenone is a novel non-steroidal MR blocker that has a dose-dependent antihypertensive effect compared with placebo.

- Esaxerenone has a good safety profile and shows no adverse events related to sex hormones.

- This study determined the optimal doses of esaxerenone for lowering blood pressure in Japanese patients with essential hypertension. 
Acknowledgements The authors would like to thank James Graham, $\mathrm{PhD}$, of Edanz Medical Writing for providing medical writing services, which were funded by Daiichi Sankyo Co., Ltd.

Funding This study was supported by Daiichi Sankyo. Daiichi Sankyo had a role in the study design, data collection, analysis and interpretation, and writing of the manuscript.

Author contributions YO and SY were responsible for the study design, and collection, analysis and interpretation of the data. SI was the principal medical expert and wrote the manuscript. HI and HR were coordinating investigators. All authors reviewed and approved the final manuscript.

\section{Compliance with ethical standards}

Conflict of interest SI, HI, and HR have received lecture fees and research funding from Daiichi Sankyo Co., Ltd. YO and SY are employees of Daiichi Sankyo Co., Ltd.

Publisher's note: Springer Nature remains neutral with regard to jurisdictional claims in published maps and institutional affiliations.

Open Access This article is licensed under a Creative Commons Attribution 4.0 International License, which permits use, sharing, adaptation, distribution and reproduction in any medium or format, as long as you give appropriate credit to the original author(s) and the source, provide a link to the Creative Commons license, and indicate if changes were made. The images or other third party material in this article are included in the article's Creative Commons license, unless indicated otherwise in a credit line to the material. If material is not included in the article's Creative Commons license and your intended use is not permitted by statutory regulation or exceeds the permitted use, you will need to obtain permission directly from the copyright holder. To view a copy of this license, visit http://creativecommons. org/licenses/by/4.0/.

\section{References}

1. MacMahon S, Peto R, Cutler J, Collins R, Sorlie P, Neaton J, et al. Blood pressure, stroke, and coronary heart disease. Part 1, Prolonged differences in blood pressure: prospective observational studies corrected for the regression dilution bias. Lancet. 1990; 335:765-74.

2. Whelton PK. Epidemiology of hypertension. Lancet. 1994; 344:101-6.

3. Schmieder RE. End organ damage in hypertension. Dtsch Arztebl Int. 2010;107:866-73.

4. Perez MI, Musini VM. Pharmacological interventions for hypertensive emergencies. Cochrane Database Syst Rev. 2008;1: CD003653.

5. Chobanian AV, Bakris GL, Black HR, Cushman WC, Green LA, Izzo JL Jr., et al. Seventh report of the Joint National Committee on prevention, detection, evaluation, and treatment of high blood pressure. Hypertension. 2003;42:1206-52.

6. Tedesco MA, Natale F, Calabrò R. Effects of monotherapy and combination therapy on blood pressure control and target organ damage: a randomized prospective intervention study in a large population of hypertensive patients. J Clin Hypertens (Greenwich). 2006;8:634-41.

7. Cushman WC, Ford CE, Cutler JA, Margolis KL, Davis BR, Grimm RH, et al. Success and predictors of blood pressure control in diverse North American settings: the antihypertensive and lipidlowering treatment to prevent heart attack trial (ALLHAT). J Clin Hypertens (Greenwich). 2002;4:393-404.

8. Shimamoto K, Ando K, Fujita T, Hasebe N, Higaki J, Horiuchi M, et al. The Japanese Society of Hypertension Guidelines for the Management of Hypertension (JSH 2014). Hypertens Res. 2014;37:253-390.

9. Marver D, Kokko JP. Renal target sites and the mechanism of action of aldosterone. Min Electrolyte Metab. 1983;9:1-18.

10. Vasan RS, Evans JC, Larson MG, Wilson PW, Meigs JB, Rifai N, et al. Serum aldosterone and the incidence of hypertension in nonhypertensive persons. N Engl J Med. 2004;351:33-41.

11. Gordon RD, Ziesak MD, Tunny TJ, Stowasser M, Klemm SA. Evidence that primary aldosteronism may not be uncommon: $12 \%$ incidence among antihypertensive drug trial volunteers. Clin Exp Pharm Physiol. 1993;20:296-8.

12. Gordon RD, Stowasser M, Tunny TJ, Klemm SA, Rutherford JC. High incidence of primary aldosteronism in 199 patients referred with hypertension. Clin Exp Pharm Physiol. 1994;21: $315-8$.

13. Umpierrez GE, Cantey P, Smiley D, Palacio A, Temponi D, Luster K, et al. Primary aldosteronism in diabetic subjects with resistant hypertension. Diabetes Care. 2007;30:1699-703.

14. Goodfriend TL, Egan BM, Kelley DE. Plasma aldosterone, plasma lipoproteins, obesity and insulin resistance in humans. Prostaglandins Leukot Ess Fat Acids. 1999;60:401-5.

15. Shibata H, Itoh H. Mineralocorticoid receptor-associated hypertension and its organ damage: clinical relevance for resistant hypertension. Am J Hypertens. 2012;25:514-23.

16. Epstein M, Duprez DA. Resistant hypertension and the pivotal role for mineralocorticoid receptor antagonists: a clinical update 2016. Am J Med. 2016;129:661-6.

17. Takahashi S, Katada J, Daida H, Kitamura F, Yokoyama K. Effects of mineralocorticoid receptor antagonists in patients with hypertension and diabetes mellitus: a systematic review and metaanalysis. J Hum Hypertens. 2016;30:534-42.

18. Narayan H, Webb DJ. New evidence supporting the use of mineralocorticoid receptor blockers in drug-resistant hypertension. Curr Hypertens Rep. 2016;18:34.

19. Hirsch JS, Drexler Y, Bomback AS. Aldosterone blockade in chronic kidney disease. Semin Nephrol. 2014;34:307-22.

20. Whelton PK, Carey RM. The 2017 American College of Cardiology/American Heart Association clinical practice guideline for high blood pressure in adults. JAMA Cardiol. 2018;3: 352-3.

21. Williams B, Mancia G, Spiering W, Rosei EA, Azizi M, Burnier $\mathrm{M}$, et al. $2018 \mathrm{ESC} / \mathrm{ESH}$ Guidelines for the management of arterial hypertension. Eur Heart J. 2018;39:3021-104.

22. Weinberger MH, Roniker B, Kraus SL, Weiss RJ. Eplerenone, a selective aldosterone blocker, in mild-to-moderate hypertension. Am J Hypertens. 2002;15:709-16.

23. Selera tablets $25 \mathrm{mg} /$ Selera tablets $50 \mathrm{mg} /$ Selera tablets $100 \mathrm{mg}$ [package insert 2007]. Tokyo: Pfizer Japan Inc. [in Japanese].

24. Arai K, Tsuruoka H, Homma T. CS-3150, a novel non-steroidal mineralocorticoid receptor antagonist, prevents hypertension and cardiorenal injury in Dahl salt-sensitive hypertensive rats. Eur J Pharm. 2015;769:266-73.

25. Arai K, Morikawa Y, Ubukata N, Tsuruoka H, Homma T. CS3150 , a novel nonsteroidal mineralocorticoid receptor antagonist, shows preventive and therapeutic effects on renal injury in deoxycorticosterone acetate/salt-induced hypertensive rats. J Pharm Exp Ther. 2016;358:548-57.

26. Kato M, Furuie H, Shimizu T, Miyazaki A, Kobayashi F, Ishizuka H. Single- and multiple-dose escalation study to assess pharma- 
cokinetics, pharmacodynamics and safety of oral esaxerenone in healthy Japanese subjects. Br J Clin Pharm. 2018;84:1821-9.

27. Krum H, Nolly H, Workman D, He W, Roniker B, Krause S, et al. Efficacy of eplerenone added to renin-angiotensin blockade in hypertensive patients. Hypertension. 2002;40:117-23.

28. Nishizaka MK, Zaman MA, Calhoun DA. Efficacy of low-dose spironolactone in subjects with resistant hypertension. Am J Hypertens. 2003;16(11 Pt 1):925-30.
29. White WB, Duprez D, St Hillaire R, Krause S, Roniker B, KuseHamilton J, et al. Effects of the selective aldosterone blocker eplerenone versus the calcium antagonist amlodipine in systolic hypertension. Hypertension. 2003;41:1021-6.

30. Williams GH, Burgess E, Kolloch RE, Ruilope LM, Niegowska J, Kipnes MS, et al. Efficacy of eplerenone versus enalapril as monotherapy in systemic hypertension. Am J Cardiol. 2004;93: 990-6. 